\title{
ADAR2 editing activity in newly diagnosed versus relapsed pediatric high-grade astrocytomas
}

\author{
Sara Tomaselli ${ }^{1}$, Federica Galeano ${ }^{1}$, Luca Massimi ${ }^{2}$, Concezio Di Rocco ${ }^{2}$, Libero Lauriola ${ }^{3}$, Angela Mastronuzzi ${ }^{1}$, \\ Franco Locatelli, ${ }^{1,4}$ and Angela Gallo ${ }^{1 *}$
}

\begin{abstract}
Background: High-grade (WHO grade III and IV) astrocytomas are aggressive malignant brain tumors affecting humans with a high risk of recurrence in both children and adults. To date, limited information is available on the genetic and molecular alterations important in the onset and progression of pediatric high-grade astrocytomas and, even less, on the prognostic factors that influence long-term outcome in children with recurrence. A-to-I RNA editing is an essential post-transcriptional mechanism that can alter the nucleotide sequence of several RNAs and is mediated by the ADAR enzymes. ADAR2 editing activity is particularly important in mammalian brain and is impaired in both adult and pediatric high-grade astrocytomas. Moreover, we have recently shown that the recovered ADAR2 activity in high-grade astrocytomas inhibits in vivo tumor growth. The aim of the present study is to investigate whether changes may occur in ADAR2-mediated RNA editing profiles of relapsed high-grade astrocytomas compared to their respective specimens collected at diagnosis, in four pediatric patients.

Methods: Total RNAs extracted from all tumor samples and controls were tested for RNA editing levels (by direct sequencing on CDNA pools) and for ADAR2 mRNA expression (by qRT-PCR).

Results: A significant loss of ADAR2-editing activity was observed in the newly diagnosed and recurrent astrocytomas in comparison to normal brain. Surprisingly, we found a substantial rescue of ADAR2 editing activity in the relapsed tumor of the only patient showing prolonged survival.

Conclusions: High-grade astrocytomas display a generalized loss of ADAR2-mediated RNA editing at both diagnosis and relapse. However, a peculiar Case, in complete remission of disease, displayed a total rescue of RNA editing at relapse, intriguingly suggesting ADAR2 activity/expression as a possible marker for long-term survival of patients with high-grade astrocytomas.
\end{abstract}

Keywords: High-grade astrocytomas, RNA editing, ADAR2

\section{Background}

Astrocytoma grade III (anaplastic astrocytoma, AA) and astrocytoma grade IV (glioblastoma multiforme, GBM) are malignant, highly aggressive human brain tumors, characterized by an intrinsic tendency to recur. The median overall survival (OS) time after diagnosis is $12-18$ months in both children and adults and decreases to a few months for patients with recurrence [1,2]. Despite multimodal treatment approaches, including extensive surgical resection and innovative radio- and chemotherapies,

\footnotetext{
* Correspondence: angela.gallo@opbg.net

'Laboratory of RNA Editing, Department of Pediatric Haematology/Oncology, Bambino Gesù Children's Hospital, IRCCS, Piazza S. Onofrio 4, Rome 00165, Italy

Full list of author information is available at the end of the article
}

the outcome for patients with high-grade astrocytomas has not significantly improved over time. Of note, available data suggest that very young children (age $<3$ years) have a more favorable prognosis than older patients with similar tumors, even if recurrence is common also in this subset of patients [3].

Differently from adults in which malignant astrocytomas are the most frequent primary brain tumors, the pediatric counterparts account for only $6-12 \%$ of all brain neoplasms $[4,5]$. Consequently, to date limited information is available on the genetic and molecular alterations in pediatric patients important for the onset and progression of high-grade astrocytomas and even less is known about

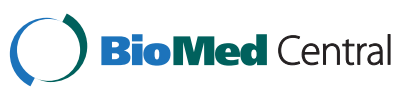


the prognostic factors that influence the long-term outcome in children with recurrence [5-7].

A-to-I RNA editing is an essential post-transcriptional mechanism that changes Adenosine (A) into Inosine (I) within RNA molecules due to the action of the ADAR (adenosine deaminase acting on dsRNA) enzymes. As Inosine is "read" as Guanosine by the splicing and translation machineries, the ADAR enzymes can generate a variety of RNAs and proteins different from those genetically coded. Three ADAR enzymes exist in mammals: ADAR1 and ADAR2 are ubiquitously expressed and catalytically active, whilst ADAR3 shows brain-specific expression and is enzymatically inactive [8,9]. ADARs can bind RNA targets through their RNA-binding domains (RBDs) and convert A into I thanks to the highly conserved deaminase domain (DM) $[8,9]$. RNA editing levels depend on the different substrates/sites, cell types, tissues and developmental stage [10,11]. $\operatorname{Adarl}^{-1-}$ and Adar2 $2^{-/-}$knockout mice die at embryonic or post-natal stages, respectively, indicating that these enzymes are essential for survival in mammals $[12,13]$.

Compared to other tissues the mammalian brain carries the highest amount of inosines [14]. Indeed several edited transcripts have been identified in the central nervous system (CNS), where ADAR2 seems to play a major role [12]. Some transcripts, coding for proteins important for a correct brain development and function, undergo editing events that change amino acid sequence (recoding editing) in crucial positions for protein activity, such as the receptor subunits of the AMPA glutamategated ion channel (GluR-B, $-\mathrm{C},-\mathrm{D})$, the Kainate receptors (GluR-5, GluR-6), the potassium voltage-gated channel (Kv1.1), the serotonin receptor $\left(5-\mathrm{HT}_{2 \mathrm{C}} \mathrm{R}\right)$ and the $\alpha 3$ subunit of the $\gamma$-amino butyric acid (GABA) receptor [9]. Interestingly, it has been shown that glutamate receptor antagonists inhibit in vitro proliferation of several human tumor cells, including gliomas [15] and that silencing of a specific AMPA receptor subunit reduces glioma growth in vivo [16]. Furthermore, it has been demonstrated that editing events within GluR-B inhibits glioma cell migration in vivo [17].

In view of these data, it is not surprising that alterations in A-to-I RNA editing in these transcripts have been observed in several human diseases affecting the CNS, including brain tumors [9]. In particular, a generalized hypoediting in both adult and pediatric high-grade astrocytomas when compared to normal brain tissues has been observed [9,18-21]. Moreover, we have recently demonstrated that the rescue of ADAR2 activity in astrocytoma cells prevents tumor growth in vivo, through the modulation of a specific molecular pathway involved in the cell cycle G1/S checkpoint [22].

The aim of the present study is to analyse ADAR2mediated RNA editing profiles in four pediatric matched pairs of high-grade astrocytomas collected at the time of diagnosis and at recurrence, in order to investigate whether changes occur throughout disease progression.

\section{Methods}

\section{Patients and samples collection}

Four pediatric patients with high-grade astrocytomas, similar tumor location and local recurrences were enrolled in this study. The patients' clinical data are summarized in Table 1.

The matched tumor samples were dissected from the proliferative core of the tumors and split in two halves, with one half fixed in $10 \%$ formalin for immunohistochemistry (IHC) analysis and the second half stored at $-80^{\circ} \mathrm{C}$ for molecular studies. Non-tumoral white matter samples (a pool of two), isolated from the same brain area of the tumors and obtained from pediatric patients undergoing focal brain resection for head injury sequelae (e.g. brain contusion), were used as normal control after being anonymized.

The study was revised and approved by the Institutional Review Board (IRB) of the local committee (Bambino Gesù Children's Hospital, Rome) on the use of human samples for experimental studies. Informed consent was obtained from all the patients' parents to the use of biological samples for research purposes.

\section{Editing analysis}

For RNA editing analysis, total RNA was isolated from tumor and control brain tissues with TRIzol reagent (Invitrogen, Carlsbad, CA, USA) according to the manufacturer's instructions. Total RNAs were treated with DNAse and cDNAs were generated using the ImProm-II Reverse Transcription System (Promega, Madison, WI, USA) and random hexamers or transcript-specific oligonucleotides (available on request). Three independent RT-PCRs (reverse transcriptase-polymerase chain reactions) were performed for each sample. Direct sequencing (ABI 3500 Genetic Analyzer, Applied Biosystems, Foster City, USA) was performed on cDNA pools and the editing levels at specific sites were measured as previously described $[20,23]$. Briefly, in the sequence chromatogram the Adenosine nucleotide that undergoes editing appears as a double peak: Adenosine for the unedited forms and Guanosine for those edited (the height of the two peaks was used for calculation of editing percentage) (Additional file 1: Figure S1).

\section{Analysis of mRNA expression levels}

Gene-specific exon-exon boundary PCR products (TaqMan gene expression assays, Applied Biosystems) were measured by means of a PE Applied Biosystems PRISM 7700 sequence detection system during 40 cycles. $\beta$-actin mRNA was used for normalization and 
Table 1 Clinical features of four children with high-grade astrocytoma

\begin{tabular}{|c|c|c|c|c|}
\hline & Case 1 & Case 2 & Case 3 & Case 4 \\
\hline$\overline{\text { Sex }}$ & $\mathrm{F}$ & M & M & $\mathrm{F}$ \\
\hline Age at diagnosis & $\leq 13$ years & $\leq 12$ years & $\leq 8$ years & $\leq 3$ years \\
\hline \multicolumn{5}{|c|}{ Newly diagnosed tumor } \\
\hline Tumor location & P left & F left & FTP right & FP left \\
\hline Resection & GTR & GTR & GTR & GTR \\
\hline Histology & GBM & GBM & AA & GBM \\
\hline Ki-67 (IHC) & $>10 \%$ & $50 \%$ & $7-10 \%$ & $60 \%$ \\
\hline Pre-radiation $\mathrm{CT}$ & / & / & / & infant protocol $(*)$ \\
\hline RT doses & 54 Gys plus TMZ & 54 Gys plus TMZ & 54 Gys plus TMZ & 59 Gys (at time of 3 year old) \\
\hline Post-radiation CT & TMZ (6 courses) & TMZ (6 courses) & TMZ (6 courses) & / \\
\hline \multicolumn{5}{|l|}{ Recurrent tumor } \\
\hline DFS (months) & 8 & 14 & 33 & 22 \\
\hline Recurrence & local & local & local & local \\
\hline Resection & GTR & GTR & GTR & GTR \\
\hline Histology & GBM & GBM & $\mathrm{AA}$ & GBM \\
\hline Ki-67 (IHC) & $40 \%$ & $50 \%$ & $60 \%$ & $50 \%$ \\
\hline Adjuvant CT & TMZ /PCV (1 course) $)^{\circ}$ & TMZ /PCV (4 courses) ${ }^{\circ}$ & TMZ /PCV (6 courses) $)^{\circ}$ & TMZ /irinotecan ( 12 courses) \\
\hline Outcome & dead & dead & dead & alive \\
\hline LPS score & / & / & / & 90 \\
\hline Disease & / & / & / & CR; off therapy \\
\hline OS (months) & 10 & 26 & 40 & 57 \\
\hline
\end{tabular}

P Parietal, F Frontal, FTP Fronto-temporo-parietal, FP Fronto-parietal, GTR Gross Total Resection, GBM Glioblastoma, AA Anaplastic Astrocytoma, IHC immunohistochemistry, RT Radiotherapy, CT Chemotherapy, TMZ Temozolomide, DFS Disease Free Survival, PCV Procarbazine-Lomustine-Vincristine, LPS score Lansky performance score (from 100 to 0 , with $100=$ healthy status), $C R$ Complete Remission, OS Overall Survival.

Until progression and death.

*Infant protocol according to the National Therapeutic Indications for infant with GBM: Methotrexate and Vincristine (1 course), Etoposide (1 course), cyclophosphamide and Vincristine (1 course), thiotepa ( 2 courses) followed by stem cell auto-grafting.

relative quantification of gene expression according to the $2^{-\Delta \Delta C t}$ method. Real-time assays were repeated in triplicates from two independent RT-PCRs. The primers were supplied by Applied Biosystems: ADAR2, ID Hs00953730_m1; $\beta$-actin, ID Hs99999903_m1. The expression level of each recurrence was calculated as relative-fold increase compared to that of the corresponding newly diagnosed tumor arbitrarily set to 1 .

To test Ki-67 expression levels, we performed semiquantitative RT-PCRs directly on the total RNA isolated from tumor and control tissues. $\beta$-actin was used to normalize the RT-PCR reactions. Ki-67 levels were also evaluated by IHC on the paraffin-embedded tissues by two independent experienced neuropathologists.

\section{Results}

RNA editing in newly diagnosed versus recurrent pediatric high-grade astrocytomas

It is emerging the idea that differences in molecular characteristics can be present in newly diagnosed versus recurrent malignant high-grade astrocytomas [2,24]. We therefore investigated whether ADAR2-mediated RNA editing, found to be important in astrocytomas, may vary throughout disease progression in four pediatric patients with supratentorial recurrent high-grade astrocytomas (Table 1).

We focused on recoding editing events of transcripts that translate into brain membrane receptors or ion channels, such as the receptor subunit of the AMPA channel (GluR-B), the receptor subunits of the Kainate channel (GluR-5 and GluR-6) and the potassium channel (Kv1.1), because these sites are mainly, if not exclusively, edited by ADAR2 enzyme [12].

We analyzed editing levels of the GluR-B transcript at the $\mathrm{Q} / \mathrm{R}$ and the $\mathrm{R} / \mathrm{G}$ sites, the GluR-6 transcript at three recoded positions identified as the $I / V, Y / C$ and $Q / R$ sites, the GluR-5 transcript carrying the $\mathrm{Q} / \mathrm{R}$ edited site and the $K v 1.1$ transcript carrying the $\mathrm{I} / \mathrm{V}$ edited site. Editing levels at all these sites were also tested in normal white matter tissues used as control and dissected from the same area of the brain where the tumors developed.

RNA editing analysis of tumor samples at diagnosis showed a significant loss of ADAR2 activity when compared with control tissues at all the sites analyzed 
(Table 2), as expected from previous studies [19-21]. Additionally, when we compared the editing profiles of newly diagnosed tumors with the corresponding relapses, we observed a generalized further loss of editing levels, with some editing sites showing a statistically significant decrease in the relapsed tumors compared with the previous lesions: the GluR-6 Y/C site $(\mathrm{p} \leq 0.05)$ and the GluR-5 Q/R site $(\mathrm{p} \leq 0.05)$ of Case 1 , the GluR-B R/G site $(\mathrm{p} \leq 0.05)$ of Case 2 and the GluR-B Q/R site $(\mathrm{p} \leq 0.01)$ of Case 3 (Figure 1 and Table 2).

Unexpectedly, the recurrence of the youngest patient (Case 4, age at diagnosis $\leq 3$ years; Table 1 ) displayed a completely different RNA editing profile in comparison to the tumor at diagnosis, showing significantly higher editing levels at all the analyzed sites (Figure 1 and Table 2).

\section{In vivo rescue of ADAR2 RNA editing activity}

Considering the surprising results observed in the recurrence of Case 4, we decided to analyze in this patient additional recoding editing sites previously found to be edited, mainly or partially, by ADAR2. We performed RNA editing analysis of the Gabra-3 I/M site (edited by both ADAR1 and ADAR2) [25], the BLCAP Y/C, Q/R sites (edited by both ADAR enzymes) and the K/R site (edited mainly by ADAR2) [26,27] in the tumor tissues of Case 4 and controls.

Editing within the Gabra-3 transcript controls trafficking of $\alpha 3$-containing receptors to the cell membrane [28]. Despite the fact that the role of editing events within $B L C A P$ are still unknown, it has been proposed that this protein is a novel prognostic biomarker in bladder cancer and it is associated with cell proliferation [29].

This further analysis confirmed a rescue of RNA editing levels in the relapse of Case 4 for all the tested sites, with editing values similar to those found in normal brain (Figure 2A). Of note, the only site of BLCAP transcript showing a significant editing rescue was the
$\mathrm{K} / \mathrm{R}$ site, which is the only one mainly modified by ADAR2 [26].

In order to rule out any possible unintentional contamination of non-tumor tissue in the relapse of Case 4, we measured the levels of Ki-67 cell proliferation index directly on the RNA samples used for the RNA editing molecular assays (Figure 2A). As expected for neoplastic tissues, both the newly diagnosed and recurrent tumor samples of Case 4 showed over-expression of Ki-67 mRNA when compared with normal white matter (Figure 2B-C). A similar result on the same samples was obtained by IHC analysis (Table 1). High Ki-67 levels were also detected by semi-quantitative RT-PCR (data not shown) and IHC (Table 1) in the tumor tissues of Cases 1-2-3.

\section{ADAR2 expression levels in pediatric high-grade astrocytomas}

ADAR2 is the enzyme mainly responsible for the recoding editing at the sites analyzed in this study $[12,20]$. Therefore, we investigated whether fluctuation in ADAR2 mRNA occurred in tumor samples that may partially explain the editing profiles of the all Cases reported.

We found a significant decrease of $A D A R 2$ expression in the recurrences of Cases 1-3 when compared to their newly diagnosed tumors (Figure 3 ). On the contrary, a significant higher ADAR2 expression level was found in the relapse of Case 4 when compared with the tumor at diagnosis (Figure 3), which can correlate with the rescued editing profiles found in the recurrence of this patient (Figure 2A).

\section{Discussion}

High-grade astrocytomas are very aggressive brain tumors, with GBM (or astrocytoma grade IV) being one of the most lethal tumors in humans. Despite the novel and aggressive surgical/therapeutic approaches, after a short period of remission these tumors frequently recur, with a median survival, after recurrence, of only few months [2]. The molecular mechanisms involved in the

Table 2 ADAR2 edited sites and their relative percentage of editing

\begin{tabular}{|c|c|c|c|c|c|c|c|c|c|}
\hline \multirow{2}{*}{$\begin{array}{l}\text { ADAR2 edited sites } \\
\text { (\% of editing } \pm \text { s.e.m.) }\end{array}$} & \multirow{2}{*}{$\begin{array}{c}\text { WM } \\
\text { (Ctrls) }\end{array}$} & \multicolumn{2}{|c|}{ Case 1} & \multicolumn{2}{|c|}{ Case 2} & \multicolumn{2}{|c|}{ Case 3} & \multicolumn{2}{|c|}{ Case 4} \\
\hline & & $N$ & $\mathbf{R}$ & $\mathrm{N}$ & $\mathbf{R}$ & $N$ & $\mathbf{R}$ & $\mathrm{N}$ & $\mathrm{R}$ \\
\hline GluR-B Q/R & $100( \pm 0)$ & $90.3( \pm 5.6)$ & $92.1( \pm 0.3)$ & $87.6( \pm 1.8)$ & $83.5( \pm 0.9)$ & $98.15( \pm 1.1)$ & $83( \pm 0.7)$ & $98.7( \pm 1.3)$ & $100( \pm 0)$ \\
\hline$R / G$ & $53.2( \pm 4.2)$ & $21.9( \pm 6.5)$ & $15.4( \pm 2)$ & $18.1( \pm 3.2)$ & $5( \pm 2.7)$ & $4.4( \pm 1.4)$ & $7.9( \pm 1.9)$ & $15.1( \pm 1.8)$ & $49.3( \pm 1.8)$ \\
\hline GluR-6 I/V & $58.1( \pm 0.7)$ & $20.5( \pm 3.3)$ & $8.3( \pm 2.5)$ & $17.1( \pm 4.8)$ & $12( \pm 1.5)$ & $0( \pm 0)$ & $2.7( \pm 2.7)$ & $12.9( \pm 1.7)$ & $55.2( \pm 2.4)$ \\
\hline $\mathrm{Y} / \mathrm{C}$ & $73.5( \pm 6.9)$ & $32.4( \pm 0.45)$ & $15.7( \pm 2)$ & $24.6( \pm 5.1)$ & $15.7( \pm 1.8)$ & $6.1( \pm 0.8)$ & $1.9( \pm 1.9)$ & $12.7( \pm 0.3)$ & $82.8( \pm 3.5)$ \\
\hline $\mathrm{Q} / \mathrm{R}$ & $74.6( \pm 0.9)$ & $8.6( \pm 8.6)$ & $3.8( \pm 3.8)$ & $24.5( \pm 0.1)$ & $19( \pm 2.7)$ & $3.4( \pm 3.4)$ & $6.4( \pm 0.5)$ & $10.3( \pm 5.3)$ & $75.9( \pm 0.9)$ \\
\hline GluR-5 Q/R & $63.8( \pm 1)$ & $28.7( \pm 0.2)$ & $22( \pm 0.9)$ & $27.6( \pm 4.6)$ & $20( \pm 2.2)$ & $16.4( \pm 2.4)$ & $21.1( \pm 1.2)$ & $35.9( \pm 4.9)$ & $71.5( \pm 3)$ \\
\hline $\mathrm{Kv} 1.1 \mathrm{I} / \mathrm{V}$ & $9.6( \pm 2)$ & $0( \pm 0)$ & $0( \pm 0)$ & $0( \pm 0)$ & $0( \pm 0)$ & $0( \pm 0)$ & $0( \pm 0)$ & $0( \pm 0)$ & $10.7( \pm 1.7)$ \\
\hline
\end{tabular}

$W M$ white matter, $N$ newly diagnosed tumor, $R$ recurrent tumor.

$\%$ of editing is expressed as mean of three independent experiments \pm S.E.M. 


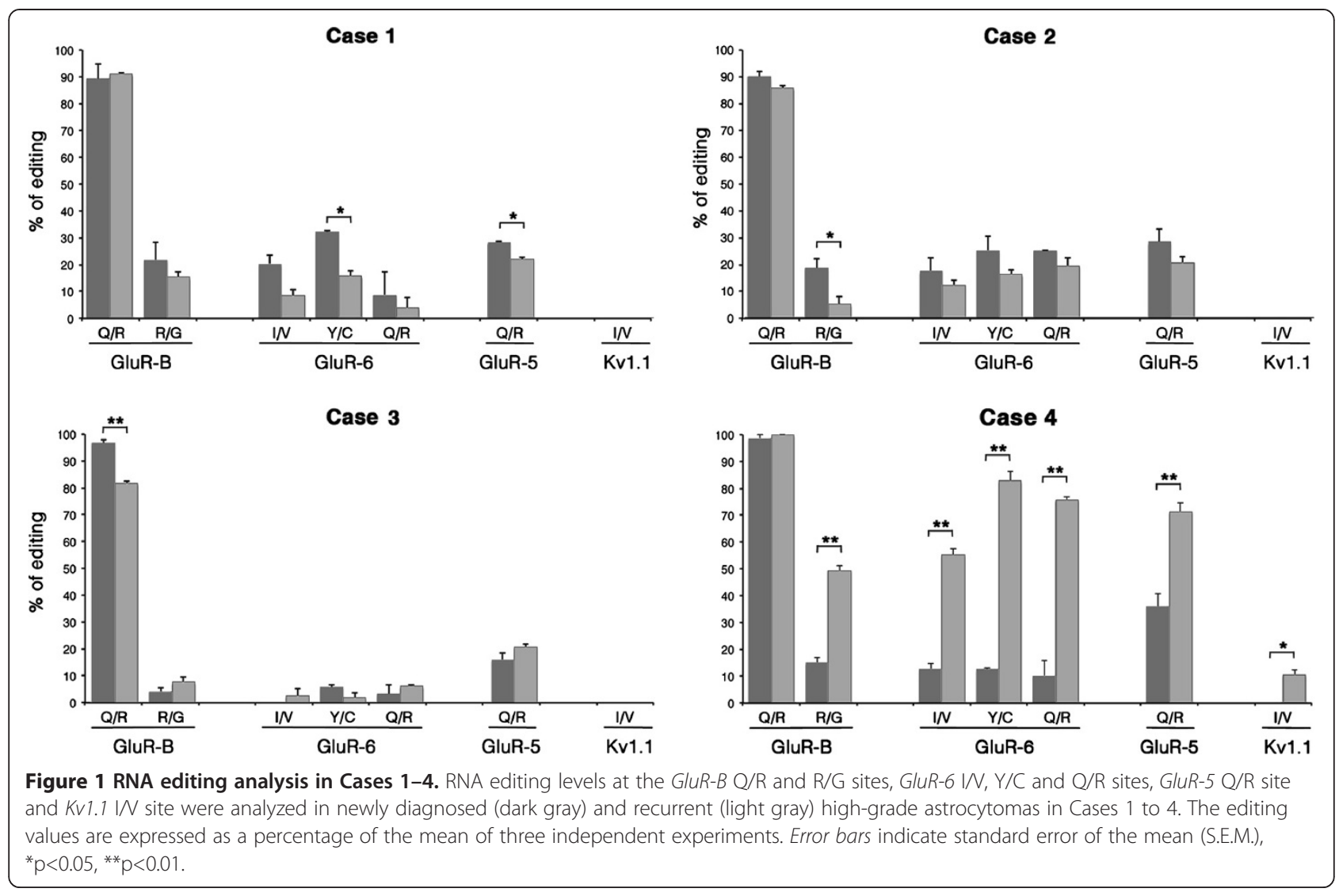

formation of malignant astrocytomas and their subsequent recurrences, as well as the signature associated with long-term survival and a positive outcome are still poorly known, especially in children, due to the rarity of these tumors during the pediatric age $[6,24,30]$.

RNA editing is an essential genetic recoding process that enhances the molecular diversity of RNAs and proteins at post-transcriptional level to different extents, depending on the cell types and tissues. In particular, ADAR2-mediated RNA editing is essential for the functional activity of many proteins expressed in the CNS from fly to mammals [9].

It has been shown that astrocytomas are characterized by a general decrease of RNA editing mediated by ADAR2 enzyme [19-22,26] and that a correlation exists between the progressive loss of ADAR2 activity and the increasing grade of tumor, with the lowest editing levels found in AAs and GBMs [20]. Furthermore, we have recently demonstrated that a recovery of ADAR2 editing activity in astrocytoma cells is necessary and sufficient to significantly inhibit tumor growth in a mouse model [22].

Considering the above findings, we investigated whether differences exist in RNA editing profiles mediated by ADAR2 between malignant high-grade astrocytomas at initial presentation and their subsequent relapse in the same patient (Table 1). To the best of our knowledge, this is the first comparative report of RNA editing analysis performed on matched pairs of newly diagnosed and recurrent tumor tissues in the same patient.

The small size of patient cohort analyzed in this study is mainly due to the rarity of high-grade astrocytomas in children, together to the difficulty in collecting tumor samples from the same patient both at diagnosis and at recurrence. Additionally, as RNA editing profiles change depending on different brain areas [20,31], we needed to collect tumor samples developed within the same brain region (supratentorial astrocytomas) from different patients.

We found an overall general decrease in RNA editing levels in both newly diagnosed and relapsed tumors in 3 out of 4 cases when compared with controls (Figure 1 and Table 2), with a significant further drop of editing in the recurrences only at few specific editing sites (Figure 1 and Table 2).

These results suggest that ADAR2-mediated RNA editing, at least on the re-coding editing sites analyzed herein, is a molecular signature for high-grade astrocytomas that does not dramatically change during tumor recurrence in children.

The most surprising result was the editing profile of Case 4, the only surviving patient (Table 1). As compared to diagnosis, its relapse sample showed a recovery of RNA editing levels at all the sites tested, with values 


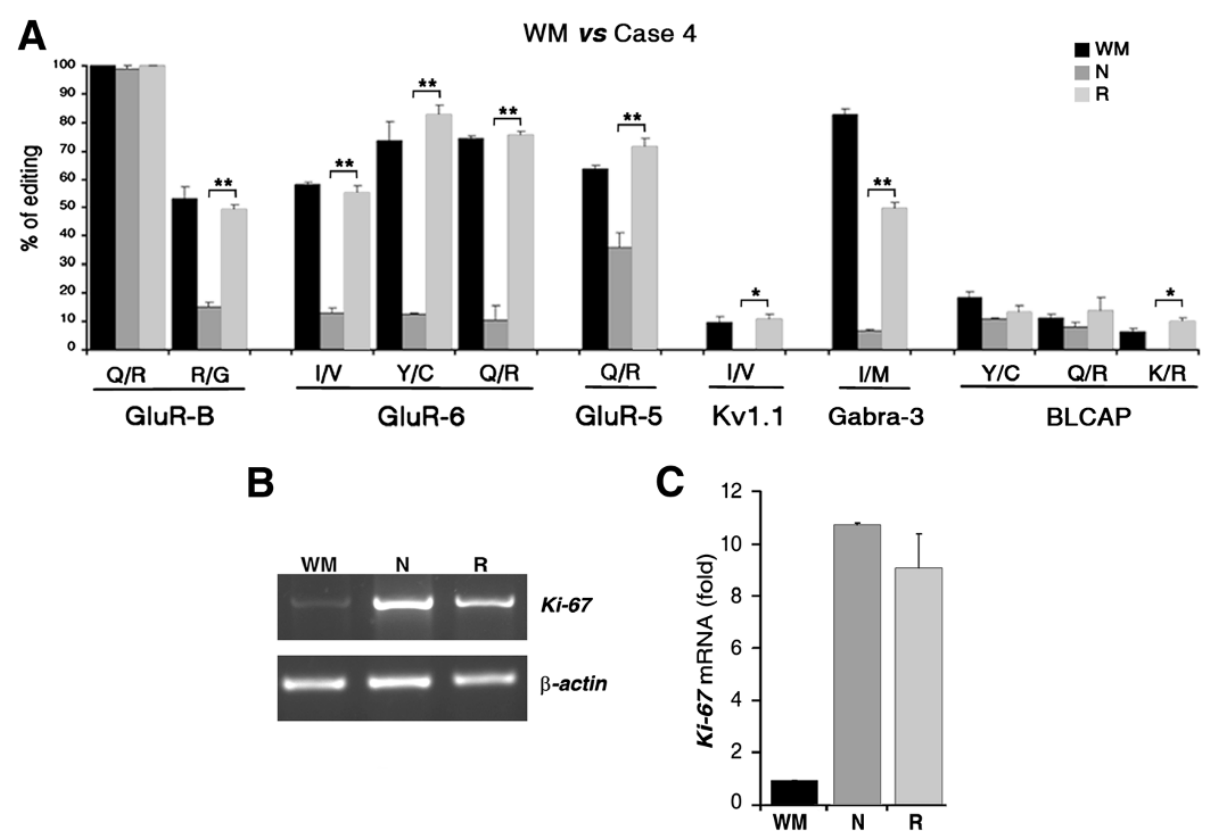

Figure 2 Comparative molecular analysis in Case 4 versus control. (A) Comparative analysis of the RNA editing levels at the G/uR-B Q/R and R/G sites, GluR-6 IN, Y/C and Q/R sites, G/uR-5 Q/R site, KV1.1 IN, Gabra-3 I/M site and BLCAP Y/C, Q/R and K/R sites among normal white matter (WM, black), newly diagnosed ( $N$, dark gray) and recurrent ( $R$, light gray) tumor tissues of Case 4 . The editing values are expressed as a percentage of the mean of three independent experiments. Error bars indicate standard error of the mean (S.E.M.), ${ }^{*} p<0.05$, ${ }^{*} p<0.01$. (B) A representative example of Ki-67 mRNA expression levels analysed by semi-quantitative RT-PCRs in the control (normal white matter), newly diagnosed and recurrent tumor samples of Case 4. (C) Densitometric analysis of Ki-67 mRNA expression is represented in arbitrary units calculated as a relative-fold increase in expression compared to the control arbitrarily set to 1. Each sample was normalized to $\beta$-actin mRNA. Error bars indicate standard error of the mean (S.E.M.) $(n=3)$.

resembling those observed in control white matter dissected from the same brain area where the tumor developed (Figure 2A). These findings were unexpected, considering that previous studies in adult and pediatric astrocytomas always reported a significant editing decrease in high-grade astrocytomas [19-22].

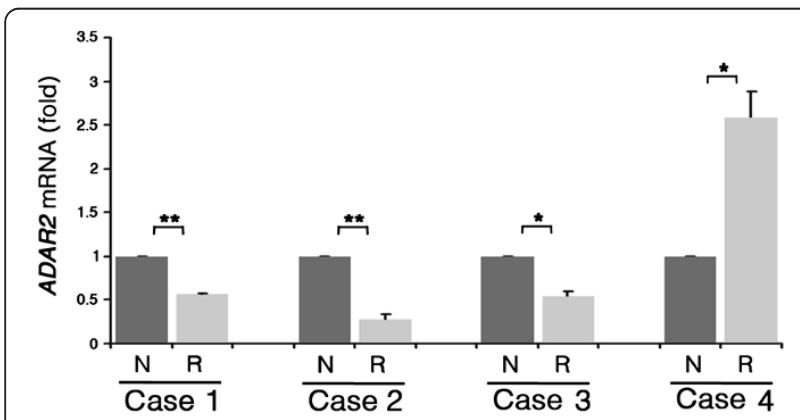

Figure 3 ADAR2 expression levels in Cases 1-4. GRT-PCR analysis of ADAR2 mRNA from newly diagnosed (dark gray) and recurrent (light gray) tumors in Cases 1 to 4 . The expression levels of each recurrence were calculated as a relative-fold increase compared to the corresponding newly diagnosed tumor arbitrarily set to 1 . Each sample was normalized to $\beta$-actin mRNA. Mean \pm s.d. $(n=2)$, ${ }^{*} p<0.05,{ }^{* *} p<0.01$.
Editing activity does not always correlate with mRNA or protein expression of ADAR enzymes [11]. According to this, a recent study showed the existence of "mediators" (i.e. proteins) that can modulate ADAR2 efficiency [32]. Nevertheless, we decided to test ADAR2 expression by qRT-PCR in our patients and only in Case 4 we found a significant increase of ADAR2 in the relapse compared to the newly diagnosed tumor (Figure 3 ). This finding correlates with the rescued editing profiles observed in the Case 4 relapsed tumor (Figure 2A). Notably, we have recently demonstrated that the forced expression of the active ADAR2 enzyme in astrocytoma cells rescues editing levels at specific sites (such as the ones tested here) and that, most importantly, this editing rescue is able to inhibit tumor growth with a significantly prolonged overall survival of mice injected with tumor cells overexpressing ADAR2 [22].

At present, little is known regarding the physiological regulation of ADAR2 expression, however it has been shown that both its expression and activity are markedly enhanced in response to glucose in pancreatic islets and beta-cells [33]. Moreover, it has been shown that in neuronal cells the cAMP response binding element (CREB), an important transcription factor, can induce ADAR2 expression [34]. The observation that infants follow a different 
protocol than older children (Table 1) is intriguing and suggest that ADAR2 expression and/or RNA editing levels could be recovered in this particular subset of patients, possibly due to specific treatments or drugs. Considering the findings of $A D A R 2$ upregulation in a peculiar Case (Case 4), we asked whether a possible correlation exist between ADAR2 mRNA expression and pediatric patient survival, interrogating available datasets. We found only a glioma array specific for pediatric patients (but not for infant) in which the clinical outcome was also reported (http://r2.amc.nl, dataset Paugh-53-MAS5.0-u133p2). We observed that, at least in this dataset, there is not a statistically significant correlation between $A D A R 2$ levels and outcome, even if a slight decrease of $A D A R 2$ expression is reported for patients died of disease compared to patients alive (data not shown).

Currently, total tumor resection, aggressive treatment and diagnosis at a younger age have been associated with longer survival of pediatric patients with high-grade astrocytomas $[6,35]$. Thus, it is intriguing to speculate that in very young children high-grade astrocytomas may be biologically different $[3,36]$. The hypothesis that younger patients (as in the Case 4 reported here) might be able to recover ADAR2 expression/activity, due to still unknown endogenous cellular factors or maybe induced by specific treatments or drugs, deserves additional investigations.

Furthermore, it would be worth considering the role of ADAR2 activity/expression as possible marker for long-term survival of patients with recurrent high-grade astrocytomas.

\section{Conclusions}

Despite the low number of paired samples investigated, RNA editing mediated by ADAR2 seems to further decrease significantly only at few specific sites throughout disease progression. Moreover, our findings relative to one peculiar Case (age $\leq 3$ years) suggest that ADAR2 activity can be rescued in vivo in tumor cells, raising the intriguing possibility that editing recovery may have contribute to the favorable outcome of this patient, as suggested by mouse model studies (22).

\section{Additional file}

Additional file 1: Figure S1. Editing levels of GluR-5 substrate in control brain tissue and Case 4. Sequence chromatograms of GluR-5 substrate using RNA extracted from control white matter (WM), Case 4 newly diagnosed GBM $(N)$ and recurrence $(R)$. The $Q / R$ edited site is represented as a double peak (adenosine plus guanosine) and is indicated by arrows.

Competing interests

The authors declare that neither financial nor non-financial competing interests exist.

\section{Authors' contributions}

ST and FG carried out the molecular genetic studies and drafted the manuscript. LM, CDR, FL and AM provided the tumor samples as well as the clinical details of the patients and revised the manuscript. LL performed the histological analysis of the tumor samples. AG designed the study, analyzed the data and wrote the manuscript. All authors read and approved the final manuscript.

\section{Acknowledgements}

This work was supported by the IG grant of AIRC (Milan, Italy) to A. G. and by special project $5 \times 1000$ AIRC to F. L. We thank Alekos Athanasidias for his precious support in the analysis of gene expression datasets.

\section{Author details}

'Laboratory of RNA Editing, Department of Pediatric Haematology/Oncology, Bambino Gesù Children's Hospital, IRCCS, Piazza S. Onofrio 4, Rome 00165 , Italy. ${ }^{2}$ Pediatric Neurosurgery Department, Policlinico Gemelli, Largo A. Gemelli 8, Rome 00168, Italy. ${ }^{3}$ Anatomopathology Department, Policlinico Gemelli, Largo A. Gemelli 8, Rome 00168, Italy. ${ }^{4}$ Università di Pavia, Strada Nuova 65, Pavia 27100, Italy.

Received: 9 July 2012 Accepted: 8 May 2013

Published: 22 May 2013

\section{References}

1. Zhu Y, Parada LF: The molecular and genetic basis of neurological tumours. Nat Rev Cancer 2002, 2:616-626.

2. Martinez R, Rohde V, Schackert G: Different molecular patterns in glioblastoma multiforme subtypes upon recurrence. J Neurooncol 2010, 96:321-329.

3. Sanders RP, Kocak M, Burger PC, Merchant TE, Gajjar A, Broniscer A: Highgrade astrocytoma in very young children. Pediatr Blood Cancer 2007, 49:888-893.

4. Perkins SM, Rubin JB, Leonard JR, Smyth MD, El Naqa I, Michalski JM, Simpson JR, Limbrick DL, Park TS, Mansur DB: Glioblastoma in children: a singleinstitution experience. Int J Radiat Oncol Biol Phys 2011, 80:1117-1121.

5. Broniscer AGA: Supratentorial high-grade astrocytoma and diffuse brainstem glioma: two challenges for the pediatric oncologist. Oncologist 2004, 9(2):197-206.

6. Song KS, Phi JH, Cho BK, Wang KC, Lee JY, Kim DG, Kim IH, Ahn HS, Park SH, Kim SK: Long-term outcomes in children with glioblastoma. J Neurosurg Pediatr 2010, 6:145-149.

7. Paugh BS, Qu C, Jones C, Liu Z, Adamowicz-Brice M, Zhang J, Bax DA, Coyle B, Barrow J, Hargrave D, et al: Integrated molecular genetic profiling of pediatric high-grade gliomas reveals key differences with the adult disease. J Clin Oncol 2010, 28:3061-3068.

8. Nishikura $\mathrm{K}$ : Functions and regulation of RNA editing by ADAR deaminases. Annu Rev Biochem 2010, 79:321-349.

9. Gallo A, Locatelli F: ADARs: allies or enemies? The importance of A-to-I RNA editing in human disease: from cancer to HIV-1. Biol Rev Camb Philos Soc 2012, 87:95-110.

10. Bass BL: Double-stranded RNA as a template for gene silencing. Cell 2000 101:235-238.

11. Wahlstedt H, Daniel C, Enstero M, Ohman M: Large-scale mRNA sequencing determines global regulation of RNA editing during brain development. Genome Res 2009, 19:978-986.

12. Higuchi M, Maas S, Single FN, Hartner J, Rozov A, Burnashev N, Feldmeyer $D$, Sprengel R, Seeburg PH: Point mutation in an AMPA receptor gene rescues lethality in mice deficient in the RNA-editing enzyme ADAR2. Nature 2000, 406:78-81.

13. Hartner JC, Schmittwolf C, Kispert A, Muller AM, Higuchi M, Seeburg PH: Liver disintegration in the mouse embryo caused by deficiency in the RNA-editing enzyme ADAR1. J Biol Chem 2004, 279:4894-4902.

14. Morse DP, Bass BL: Detection of inosine in messenger RNA by inosinespecific cleavage. Biochemistry 1997, 36:8429-8434.

15. Rzeski W, Turski L, Ikonomidou C: Glutamate antagonists limit tumor growth. Proc Natl Acad Sci U S A 2001, 98:6372-6377.

16. De Groot JF, Piao Y, Lu L, Fuller GN, Yung WK: Knockdown of GluR1 expression by RNA interference inhibits glioma proliferation. J Neurooncol 2008, 88:121-133. 
17. Ishiuchi S, Tsuzuki K, Yoshida Y, Yamada N, Hagimura N, Okado H, Miwa A, Kurihara H, Nakazato Y, Tamura M, et al: Blockage of $\mathrm{Ca}(2+)$-permeable AMPA receptors suppresses migration and induces apoptosis in human glioblastoma cells. Nat Med 2002, 8:971-978.

18. Galeano F, Tomaselli $S$, Locatelli $F$, Gallo A: A-to-I RNA editing: The "ADAR" side of human cancer. Semin Cell Dev Biol 2012, 23:244-250.

19. Maas S, Patt S, Schrey M, Rich A: Underediting of glutamate receptor GluR-B mRNA in malignant gliomas. Proc Natl Acad Sci U S A 2001, 98:14687-14692

20. Cenci C, Barzotti R, Galeano F, Corbelli S, Rota R, Massimi L, Di Rocco C, O'Connell MA, Gallo A: Down-regulation of RNA editing in pediatric astrocytomas: ADAR2 editing activity inhibits cell migration and proliferation. J Biol Chem 2008, 283:7251-7260.

21. Paz N, Levanon EY, Amariglio N, Heimberger AB, Ram Z, Constantini S, Barbash ZS, Adamsky K, Safran M, Hirschberg A, et al: Altered adenosine-to -inosine RNA editing in human cancer. Genome Res 2007, 17:1586-1595.

22. Galeano F, Rossetti C, Tomaselli S, Cifaldi L, Lezzerini M, Pezzullo M, Boldrini R, Massimi L, Di Rocco CM, Locatelli F, Gallo A: ADAR2-editing activity inhibits glioblastoma growth through the modulation of the CDC14B/ Skp2/p21/p27 axis. Oncogene 2013, 32:998-1009.

23. Larder BA, Kohli A, Kellam P, Kemp SD, Kronick M, Henfrey RD: Quantitative detection of HIV-1 drug resistance mutations by automated DNA sequencing. Nature 1993, 365:671-673.

24. Saxena A, Shriml LM, Dean M, Ali IU: Comparative molecular genetic profiles of anaplastic astrocytomas/glioblastomas multiforme and their subsequent recurrences. Oncogene 1999, 18:1385-1390.

25. Ohlson J, Pedersen JS, Haussler D, Ohman M: Editing modifies the GABA (A) receptor subunit alpha3. RNA 2007, 13:698-703.

26. Galeano F, Leroy A, Rossetti C, Gromova I, Gautier P, Keegan LP, Massimi L, Di Rocco C, O'Connell MA, Gallo A: Human BLCAP transcript: new editing events in normal and cancerous tissues. Int J Cancer 2010, 127:127-137.

27. Levanon EY, Hallegger M, Kinar Y, Shemesh R, Djinovic-Carugo K, Rechavi G, Jantsch MF, Eisenberg E: Evolutionarily conserved human targets of adenosine to inosine RNA editing. Nucleic Acids Res 2005, 33:1162-1168.

28. Daniel C, Wahlstedt H, Ohlson J, Bjork P, Ohman M: Adenosine-to-inosine RNA editing affects trafficking of the gamma-aminobutyric acid type A (GABA(A)) receptor. J Biol Chem 2011, 286:2031-2040.

29. Moreira JM, Ohlsson G, Gromov P, Simon R, Sauter G, Celis JE, Gromova I: Bladder cancer-associated protein, a potential prognostic biomarker in human bladder cancer. Mol Cell Proteomics 2010, 9:161-177.

30. Hulsebos TJ, Troost D, Leenstra S: Molecular-genetic characterisation of gliomas that recur as same grade or higher grade tumours. I Neurol Neurosurg Psychiatry 2004, 75:723-726.

31. Paupard MC, O'Connell MA, Gerber AP, Zukin RS: Patterns of developmental expression of the RNA editing enzyme rADAR2. Neuroscience 2000, 95:869-879.

32. Marcucci R, Brindle J, Paro S, Casadio A, Hempel S, Morrice N, Bisso A, Keegan LP, Del Sal G, O'Connell MA: Pin1 and WWP2 regulate GluR2 Q/R site RNA editing by ADAR2 with opposing effects. EMBO J 2011, 30:4211-4222.

33. Gan Z, Zhao L, Yang L, Huang P, Zhao F, Li W, Liu Y: RNA editing by ADAR2 is metabolically regulated in pancreatic islets and beta-cells. J Biol Chem 2006, 281:33386-33394.

34. Peng PL, Zhong X, Tu W, Soundarapandian MM, Molner P, Zhu D, Lau L, Liu S, Liu F, Lu Y: ADAR2-dependent RNA editing of AMPA receptor subunit GluR2 determines vulnerability of neurons in forebrain ischemia. Neuron 2006, 49:719-733.

35. Mahvash M, Hugo HH, Maslehaty H, Mehdorn HM, Stark AM: Glioblastoma multiforme in children: report of 13 cases and review of the literature. Pediatr Neurol 2011, 45:178-180.

36. Duffner PK, Krischer JP, Burger PC, Cohen ME, Backstrom JW, Horowitz ME, Sanford RA, Friedman HS, Kun LE: Treatment of infants with malignant gliomas: the Pediatric Oncology Group experience. J Neurooncol 1996, 28:245-256.

\section{doi:10.1186/1471-2407-13-255}

Cite this article as: Tomaselli et al:: ADAR2 editing activity in newly diagnosed versus relapsed pediatric high-grade astrocytomas. BMC Cancer 2013 13:255.

\section{Submit your next manuscript to BioMed Central and take full advantage of:}

- Convenient online submission

- Thorough peer review

- No space constraints or color figure charges

- Immediate publication on acceptance

- Inclusion in PubMed, CAS, Scopus and Google Scholar

- Research which is freely available for redistribution 